\title{
Effect of Mannitol and Sodium Chloride on Some Total Secondary Metabolites of Fenugreek Calli Cultured In vitro
}

\author{
Esam A. Hussein ${ }^{* 1}$ and Esam M. Aqlan ${ }^{1}$ \\ Botany and Microbiol. Department, Faculty of Science, Al-Azhar University, Cairo, \\ Egypt
}

Key words: Trigonella foenum-gaecum, Callus, Abiotic stress, Secondary metabolites

\begin{abstract}
Hypocotyls of Fenugreek (Trigonella foenum-graecum L.) were cut into $5 \mathrm{~mm}$ long segments and used as explants. For obtaining callus under various water and salt stress levels, MS supplemented with $2.0 \mathrm{mg} / \mathrm{l} \mathrm{BA}$ and $1.0 \mathrm{mg} / \mathrm{l}$ NAA was prepared; then divided into nine portions, the first of which was kept as control while to the other portions mannitol $(0.1$ and $0.3 \% \mathrm{w} / \mathrm{v})$ and sodium chloride $(0.1$ and $0.3 \% \mathrm{w} / \mathrm{v}$ ) were added separately and interacted. Callus formation was allowed to take place for a period of six weeks in the dark at $25^{\circ} \mathrm{C}$. Calli from various treatments were then harvested and subjected to growth, total phenolics, total flavonoids and total tannin contents determinations. Results of the present study have shown that the lower concentration of mannitol enhanced growth while the higher concentration enhanced growth, total phenolics and total flavonoid contents in comparison to the control. Mild salt stress which was created by the lower sodium chloride concentration reduced growth with concomitant increase in total phenolics, total flavonoids and total tannins while the higher salt concentration significantly decreased growth and secondary metabolite accumulation as well. Interaction treatments between mannitol and sodium chloride exhibited various effects on calli growth and secondary metabolites levels. This study throws some light on the behavior of cultured fenugreek cells grown under water and salt stress.
\end{abstract}

\section{Introduction}

Improvement of phytopharmaceutics biosynthesis by in vitro cultured plant cells can be achieved by subjecting the cultured plant cells to stress factors. Exposure to environmental factors like water or salt stresses trigger many common reactions in plants. Both of them lead to oxidative stress by increasing reactive oxygen species (Apel and Hirt 2004) and osmotic stress (Bartels and Sunkar 2005) by removing water from the cytoplasm into the extracellular space. The

${ }^{*}$ Corresponding author. <esam431@hotmail.com> ${ }^{1}$ Biology Department, Faculty of Science, Ibb University, Ibb, Yemen. 
complexity of the plant response to salt stress may result from salt toxicity in addition to osmotic effects (Hasegawa et al. 2000). Plant cells, in response to osmotic stress, tend to accumulate sugars and compatible solutes in the cytoplasm (Chen and Murata 2002) and other osmotically active low molecular weight compounds (Roychoudury et al. 2008). Fortunately, many of the stress induced compounds are useful secondary metabolites. For example, in vitro cultured plant cells were observed to synthesize extra amounts of soluble phenolics (Grace and logan 2000), anthraquinone (Nazif et al. 2000), Flavonoids (Chutipaijit et al. 2009), indole alkaloids (Chutipaijit et al. 2009) and anthocyanin (Chan et al. 2010) in response to various abiotic stress factors. Fenugreek (Trigonella foenum-graecum L.) which is adopted in this study is a famous plant with nutritive and medicinal properties. The main objective of the present study is to improve biosynthesis of phenolics, flavonoids and tannins in fenugreek calli by growing them under the effect of water stress and salt stress which can be created in culture media by the addition of mannitol and sodium chloride in a suitable proportion.

\section{Materials and methods}

Seeds of fenugreek were surface sterilized according to Chawla (2003) by submerging the explants into a solution of $70 \%$ ethanol with continuous and gentle stirring for one min, transferring them to $100 \mathrm{ml}$ conical flask containing $20 \%$ solution of commercial sodium hypochlorite (1\% active chlorine) with continuous gentle stirring for seven min. The sterilant was decanted and the seeds were washed with three successive rinses of sterile distilled water under aseptic conditions. The seeds were then dried between two layers of sterile filter papers in a Petri dish. Using sterile scalpel the seeds were transferred to Petri dishes containing hormone free MS and were allowed to germinate for one week at room temperature. Hypocotyls of the sprouts were then cut under aseptic conditions into $0.5 \mathrm{~mm}$ long for callus initiation.

For induction of callus from $5 \mathrm{~mm}$ long hypocotyl, MS containing 3\% sucrose supplemented with $2.0 \mathrm{mg} / \mathrm{l} \mathrm{BA}$ and $1.0 \mathrm{mg} / \mathrm{l} \mathrm{NAA}$ was used. The culture medium was then divided into nine portions. Water stress was created in the culture medium by the addition of 0.1 or $0.3 \%$ mannitol. Salt stress was created in the culture medium by the addition of 0.1 or $0.3 \%$ sodium chloride. Interaction treatments between mannitol and sodium chloride were also prepared. $\mathrm{pH}$ value of the culture media was adjusted to 5.7 prior to autoclaving with $0.8 \%(\mathrm{w} / \mathrm{v})$ agar (Glaxo, bacteriological grade). Autoclave sterilization $\left(15 \mathrm{~min}, 121^{\circ} \mathrm{C}\right.$ and a

pressure equal to 1.5 atmosphere) was then carried out. Culture media were dispensed into sterile $9 \mathrm{~cm}$ diam Petri dishes. Each treatment was represented by 
six replicates and each replicate contained three hypocotyls explants. The cultures were incubated in the dark at room temperature for six weeks.

The amounts of phenolics in extract of the control (non stressed callus) and calli produced under various water and salt stress conditions were determined according to the method of Spanos and Wrolstad (1990) using Folin-Ciocalteu reagent. A known weight callus was macerated in $80 \%$ ethanol and extracted to exhaustion. The extract was then dried and a known weight of the extract was dissolved in $10 \mathrm{ml}$ methanol. To $5 \mathrm{ml}$ of each sample $0.25 \mathrm{ml}$ of $10 \%$ diluted Folin-Ciocalteu reagent was added and the resulting mixture was incubated at $45^{\circ} \mathrm{C}$ for $15 \mathrm{~min}$. Absorbance of the resulted color was measured spectrophotometrically at $765 \mathrm{~nm}$. A standard curve was made using different concentrations of gallic acid and the concentration in the tested extracts were expressed as mg gallic acid equivalent per g dry callus material (mg GAE/g $\mathrm{dw})$.

Aluminium chloride colorimetric method described by Chang et al. (2002) was used for determination of total flavonoids as mg quercetin equivalent. In a clean dry test tube (three replicates), the following were mixed: $0.1 \mathrm{ml}$ of callus extract in methanol $+1.5 \mathrm{ml}$ methanol $+0.1 \mathrm{ml}$ of $10 \% \mathrm{AlCl}_{3}+0.1 \mathrm{ml}$ of $1 \mathrm{M}$ $\mathrm{CH}_{3} \mathrm{COOK}+2.8 \mathrm{ml}$ distilled water. The mixture was then kept at room temperature for $1 / 2 \mathrm{hr}$ until complete yellow color development was achieved; then absorbance of the reaction mixture was measured at $415 \mathrm{~nm}$. Concentration of total flavonoids in the callus extract was expressed as qurcetin equivalent from the standard curve made using concentrations of qurcetin from 20 to $100 \mathrm{mg} / \mathrm{l}$ in methanol.

Total tannins were determined gravimetrically according to Ali et al. (1991). One $\mathrm{g}$ fresh callus was boiled for one hr with two successive quantities, each of $100 \mathrm{ml}$ of acetone: water $(1: 1)$. Filtration was carried out and the combined filtrates were completed to $250 \mathrm{ml}$ using distilled water and heated again till boiling. Tannins were then precipitated from the extract as copper tannate by the addition of $30 \mathrm{ml}$ of $15 \%$ aqueous solution of copper acetate. The precipitate was collected on ashless filter paper, washed with water till free from copper acetate, ignited in porcelain crucible that was previously ignited to a constant weight. A few drops of nitric acid were added to the residue and reignited to a constant weight. The weight of the resulting copper oxide was determined and the amount of tannins was calculated.

Statistical analysis was performed using SPSS statistical package software version 15 (USA). The results are presented as mean \pm standard error and analyzed with T-test. The data were considered significant when $p$ value was < 0.05 . 


\section{Results and Discussion}

Results of the present study as illustrated in Tables $1-2$ and Fig. 1 have shown that mannitol improved callus growth as measured by fresh and dry weight biomass. Maximum dry weight (Table 2) was obtained with $0.1 \%$ mannitol and reached up to 0.068 gm compared to 0.044 gm recorded with control callus. Salt treatment generally significantly reduced growth as measured by either fresh or dry callus biomass. The effect was much more pronounced with the higher salt concentration where the dry weight of callus decreased to $0.027 \mathrm{~g}$ (Table 2). As regards to interaction between mannitol and sodium chloride, it can be observed also that $0.1 \%$ mannitol could alleviate the negative effects of sodium chloride on growth and even improve growth significantly over control calli (Tables 1 and 2).

Table 1. Effect of mannitol and sodium chloride on callus fresh weight (g).

\begin{tabular}{lcccc}
\hline & & \multicolumn{3}{c}{ Sodium chloride concentration in culture media } \\
& & \multicolumn{6}{c}{$(\mathrm{w} / \mathrm{v})$} & \\
\hline Mannitol & & 0 & $0.10 \%$ & $0.30 \%$ \\
concentration & 0 & $0.494 \pm 0.002$ & $0.386 \pm 0.002^{*}$ & $0.266 \pm 0.003^{*}$ \\
in culture & $0.10 \%$ & $0.766 \pm 0.018^{*}$ & $0.600 \pm 0.003^{*}$ & $0.654 \pm 0.004^{*}$ \\
media $(\mathrm{w} / \mathrm{v})$ & $0.30 \%$ & $0.664 \pm 0.001^{*}$ & $0.462 \pm 0.001$ & $0.162 \pm 0.001^{*}$ \\
\hline
\end{tabular}

Each value is a mean of three determinations \pm standard error, ${ }^{*}=$ Significant value.

Table 2. Effect of mannitol and sodium chloride on callus dry weight (g).

\begin{tabular}{lcccc}
\hline & & \multicolumn{3}{c}{ Sodium chloride concentration in culture media } \\
& & \multicolumn{6}{c}{$(\mathrm{w} / \mathrm{v})$} & \\
\hline Mannitol & & 0 & $0.10 \%$ & $0.30 \%$ \\
concentration & 0 & $0.044 \pm 0.002$ & $0.034 \pm 0.001^{*}$ & $0.027 \pm 0.001^{*}$ \\
in culture & $0.10 \%$ & $0.068 \pm 0.001^{*}$ & $0.047 \pm 0.001$ & $0.048 \pm 0.001$ \\
media (w/v) & $0.30 \%$ & $0.066 \pm 0.001^{*}$ & $0.043 \pm 0.002$ & $0.031 \pm 0.003^{*}$ \\
\hline
\end{tabular}

Each value is a mean of three determinations \pm standard error ${ }^{*}=$ Significant value.

Results of the present study (Table 3) indicate that lower concentration of mannitol slightly decreased phenolics contents while the higher concentration increased total phenolics up to $45.84 \mathrm{mg} / \mathrm{g}$ dry weight which, in comparison to control $(25.33 \mathrm{mg})$, represents a significant increase. Results presented in the same table show that the lower concentration of sodium chloride (mild salt stress) slightly enhanced phenolics biosynthesis while the higher concentration significantly decreased it $(19.67 \mathrm{mg} / \mathrm{g})$. The interaction between mannitol and sodium chloride exhibited various effects on total phenolics. 


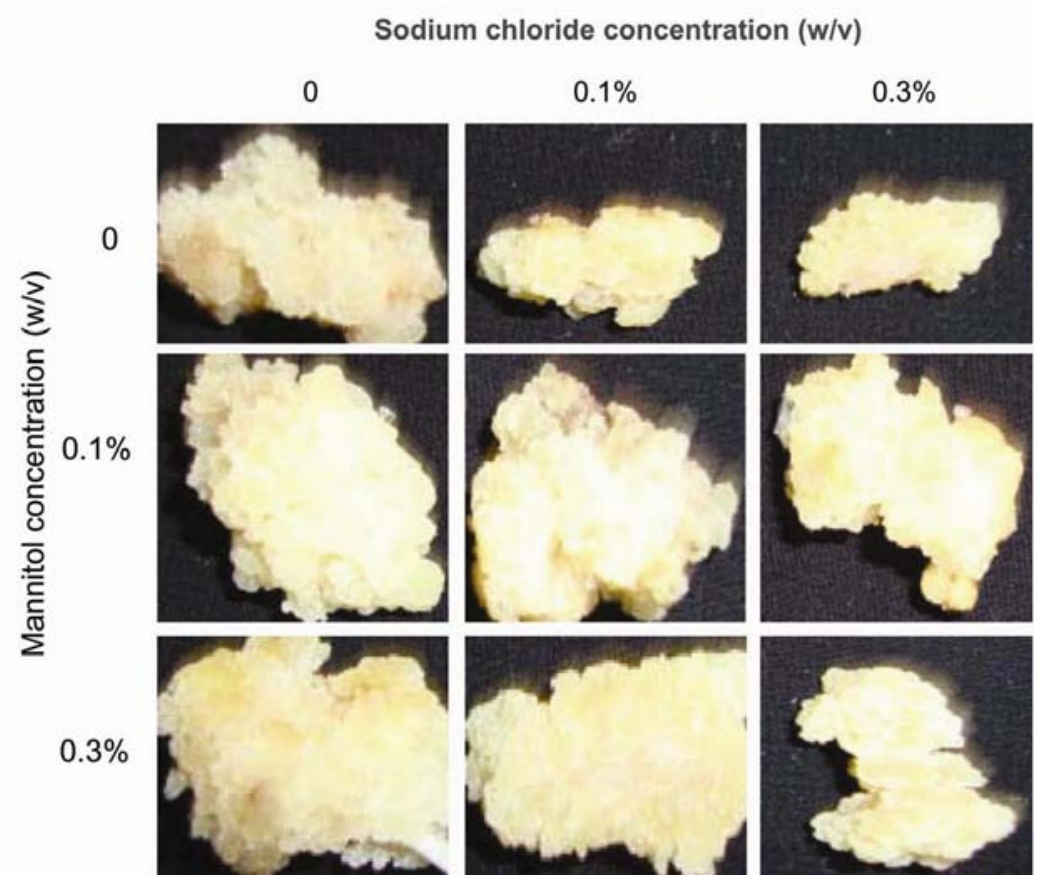

Fig. 1. Calli obtained under various mannitol and sodium chloride treatments.

Table 3. Effect of mannitol and sodium chloride on total phenolics ( $\mathrm{mg} / \mathrm{g}$ dry weight).

\begin{tabular}{lcccc}
\hline & \multicolumn{4}{c}{ Sodium chloride concentration in culture media } \\
& \multicolumn{4}{c}{$\mathrm{w} / \mathrm{v})$} \\
\hline Mannitol & & 0 & $0.10 \%$ & $0.30 \%$ \\
concentration & 0 & $25.33 \pm 0.88$ & $28.33 \pm 0.88^{*}$ & $19.67 \pm 0.67^{*}$ \\
in culture & $0.10 \%$ & $24.29 \pm 1.53$ & $29.29 \pm 1.00^{*}$ & $21.89 \pm 1.20^{*}$ \\
media $(\mathrm{w} / \mathrm{v})$ & $0.30 \%$ & $45.84 \pm 0.88^{*}$ & $30.33 \pm 0.58^{*}$ & $33.07 \pm 0.88^{*}$ \\
\hline
\end{tabular}

Each value is a mean of three determinations \pm standard error, ${ }^{*}=$ Significant value.

As regards flavonoids, results illustrated in Table 4 show that significant increases were recorded when calli were grown on culture medium containing $0.1 \%$ sodium chloride alone or combined with $0.3 \%$ mannitol where it reached up $18.300 \mathrm{mg} / \mathrm{g}$ and $17.472 \mathrm{mg} / \mathrm{g}$ alternatively in comparison to control calli $(14.467 \mathrm{mg} / \mathrm{g})$. All the other treatments exhibited either slight or significant decreases.

With respect to tannins, it seems that they were more sensitive to salt than to mannitol treatment. In comparison to control, as illustrated in Table 5, calli contents of tannins were insignificant under both 0.1 and $0.3 \%$ mannitol added to the culture media while on the other hand the lower sodium chloride concentration (mild salt stress) significantly enhanced the callus content of 
tannins where it reached up to $41.43 \mathrm{mg} / \mathrm{g}$. The higher salt concentration, in contrast, significantly lowered tannin to $12.47 \mathrm{mg} / \mathrm{g}$ compared to control (23.67 $\mathrm{mg} / \mathrm{g})$.

Table 4. Effect of mannitol and sodium chloride on flavonoids (mg/g dry weight).

\begin{tabular}{lcccc}
\hline & \multicolumn{4}{c}{ Sodium chloride concentration in culture media } \\
& & \multicolumn{6}{c}{$(\mathrm{w} / \mathrm{v})$} & \\
\hline Mannitol & & 0 & $0.10 \%$ & $0.30 \%$ \\
conc. in & 0 & $14.467 \pm 0.120$ & $18.300 \pm 0.116^{*}$ & $12.468 \pm 0.067^{*}$ \\
culture & $0.10 \%$ & $14.207 \pm 0.120$ & $10.739 \pm 0.116^{*}$ & $11.322 \pm 0.153^{*}$ \\
media (w/v) & $0.30 \%$ & $11.573 \pm 0.186^{*}$ & $17.472 \pm 0.120^{*}$ & $8.451 \pm 0.116^{*}$ \\
\hline
\end{tabular}

Each value is a mean of three determinations \pm standard error, ${ }^{*}=$ Significant value.

Table 5. Effect of sodium mannitol and sodium chloride on tannins (mg/g dry weight).

\begin{tabular}{lcccc}
\hline & \multicolumn{4}{c}{ Sodium chloride concentration in culture media } \\
& \multicolumn{4}{c}{$(\mathrm{w} / \mathrm{v})$} \\
\hline Mannitol conc. in & & 0 & $0.10 \%$ & $0.30 \%$ \\
culture media & 0 & $23.67 \pm 0.52$ & $41.43 \pm 0.49^{*}$ & $12.47 \pm 0.07^{*}$ \\
$(\mathrm{w} / \mathrm{v})$ & $0.10 \%$ & $24.54 \pm 0.41$ & $27.50 \pm 0.27^{*}$ & $23.96 \pm 0.57$ \\
& $0.30 \%$ & $22.47 \pm 0.19$ & $23.66 \pm 0.12$ & $18.36 \pm 0.12^{*}$ \\
\hline
\end{tabular}

Creating a case of osmotic or ionic stress in the culture media of plant cells in vitro is an interesting approach to secondary metabolite improvement and the effect on in vitro cultured cells may be much more pronounced than with whole plant studies due to the absence of root barrier. Vanshree et al. (2004) and Namdeo (2007) mentioned that stress factors can improve secondary metabolite biosynthesis.

The lower concentration of mannitol $(0.1 \%)$ enhanced growth as measured by the fresh (Table 1) and dry (Table 2) biomass determination but exhibited almost no significant effects on phenolics (Table 3), flavonoids (Table 4) or tannins (Table 5) while the higher concentration of mannitol $(0.3 \%)$ may have developed a case of water stress that was suitable for enhancement of phenolics and flavonoid accumulation in the cultured cells or may have enhanced sucrose levels or exhibited some effects on specific genes because in earlier studies (Hudak et al. 2010) mannitol treatment was observed to increase sucrose levels in potato calli extremely. Succrose is a key compound that can encourage many metabolic pathways. Solanelli et al. (2009) suggested that sugars play a role in regulating the genes responsible for the synthesis of secondary metabolites like polyphenolics, anthocyanins and flavonoids. El-Far and Taie (2009) also observed that sorbitol treatment resulted in an increase in sweat potato callus 
phenolics and flavonoids. In addition, Dixon and Priva (1995) reported that soluble phenolics represent the most widely distributed secondary metabolites in the plant kingdom and could be enhanced as a powerful antioxidant in plant tissues suffering from different stress factors. Mannitol stress increased osmotically active molecules like proline and polyamines in a study carried out by Tonon et al. (2004) on Fraxinus angustifolia. Chutipaijit et al. (2009) also reported that flavonoids are stimulated in stressed rice tissues to impair reactive oxygen species (ROS) induced by oxidative stress.

In the present study, mild salt stress $(0.1 \%$ sodium chloride added to the culture media) suppressed growth and enhanced phenolics, flavonoids and tannin contents as illustrated in Tables 1, 2, 3, 4 and 5, respectively. The results obtained here may agree with Lindsey (1985) who came to the conclusion that treatments which suppress cell growth and primary metabolism seem to improve capsaicin synthesis of in vitro cultured Capsicum cells and also with Nazif et al. (2000) who observed that salt stress, which adversely affected growth, stimulated anthraquinone production in cell suspension cultures of Cassia acutifolia. Adverse effects on callus growth with concomitant increase in osmotically active molecules were observed also in studies carried out by Zhao et al. (2009) and Lokhande et al. (2010).

The higher concentration of sodium chloride $(0.3 \%)$ may possibly be strong enough to adversely affect biomass, phenolics, flavonoids and tannins biosynthesis as well. Bartles and Sunkar (2005) reported that high salt concentration inhibits the activities of most enzymes because of perturbation of the hydrophilic electrostatic balance between forces maintaining protein structure.

Induction of callus formation from fenugreek hypocotyl explants under the effect of $0.1 \%$ mannitol increased callus growth but insignificantly affected levels of phenolics, flavonoids or tannins while the higher mannitol concentration $(0.3 \%)$ increased phenolics, decreased flavonoids and insignificantly affected tannins. Mild sodium chloride treatment $(0.1 \%)$ decreased growth and significantly increased the levels of phenolics, flavonoids and tannins in callus tissues while the higher salt concentration $(0.3 \%)$ significantly reduced growth, phenolics-, flavonoids- and tannins content. It seems that mannitol and sodium chloride, if added in appropriate concentration to culture media, may enhance secondary metabolites accumulation in fenugreek cells cultured in vitro and hence can be used as factors for improving secondary metabolites production in vitro. 


\section{References}

Ali AA, Ross SA, Mesbah MK and El-Moghazy SA (1991) Phytochemical study of Limonium axillare (Forssk.). Bull. Fac. Pharm. Cairo Univ. 29: 59-62.

Apel K and Hirt M (2004) Reactive oxygen species: metabolism, oxidative stress and signal transduction. Annu. Rev. Plant Biol. 55: 373-399.

Bartles D and Sunkar R (2005) Drought and salt tolerance in plants. Critical Reviews in Plant Science 24: 23-58.

Chan LK, Koay SS, Boey RL and Bhatt A (2010) Effects of abiotic stress on biomass and anthocyanin production in cell cultures of Melastoma malabathricum. Biol. Res. 43: 127135.

Chang C, Yang M, Wen H and Chern J (2002) Estimation of total flavonoid content in propolis by two complementary colorimetric methods. J. Food and Drug Analysis 10: 178-182.

Chen THH and Murata N (2002) Enhancement of tolerance of abiotic stress by metabolic engneering of betains and other compatible solutes. Curr. Opin. Plant Biol. 5: 250-257.

Chutipaijit S, Cha-Um S and Sompornpailin K (2009) Differential accumulations of praline and flavonoids in indica rice varieties against salinity. Pakistan J. Bot. 45: 2497-2506.

Dixon R and Priva N (1995) Stress induced phenyl propanoid metabolism. Plant Cell 7: 1085-1097.

El-Far MM and Taie HA (2009) Antioxidant activities, total anthocyanins, phenolics and flavonoids contents in some sweet potato genotypes under stress of different concentrations of sucrose and sorbitol. Australian J. Basic Appl. Sci. 3: 3609-3616.

Grace SC and Logan BA (2000) Energy dissipation and radical scavenging by the plant phenyl propanoid pathway. Phil. Trans. Royal Soc. B. 355: 1499-1510.

Hasegawa PM, Bressan RA, Zhu JK and Bohnert HJ (2000) Plant cellular and molecular responses to high salinity. Annu. Rev. Plant Physiol. 51: 463-499.

Hudak I, Dobranszki J, Sardi E and Hevesi M (2010) Changes in carbohydrate content of potato calli during osmotic stress induced by mannitol. Acta. Biologica Hungarica 61: 234-236.

Lindsey K (1985) Manipulation by nutrient limitation of the biosynthetic activity of immobilized cells of Capsicum frutescens Mill. Cv. Annuum. Planta 165: 126-133.

Lokhande VH, Nikam TD and Penna S (2010) Biochemical, physiological and growth changes in response to salinity in callus cultures of Sesuvium portulacastrum L. Plant Cell Tiss. Org. Cult. 102: 17-25.

Namdeo AG (2007) Plant cell elicitation for production of secondary metabolites. Pharmacognosy Rev. 1: 69-79.

Nazif NM, Rady MR and Seif El-Nasr MM (2000) Stimulation of anthraquinone production in suspension cultures of Cassia acutifolia by salt stress. Fitoterapia 71: 3440.

Roychoudry A, Basu S, Sarka SN and Sengupta DN (2008) Comparative physiological and molecular responses of common aromatic indica rice cultivars to high salinity with non aromatic indica rice cultivars. Plant Cell Rep. 27: 1395-1410. 
Solanelli C, Allesandra P, Elena L, Amedeo A and Pierdominco P (2006) Sucrose specific induction of the anthocyanin biosynthetic pathway in Arabidopsis. Plant Physiol. 140: 637-646.

Spanos GA and Wrolstad RE (1990) Influence of processing and storage on the phenolic composition of Thompson seedless grape juice. J. Agricul. Food Chem. 38:1565-1571.

Tonon G, Kevers C, Faire-Rampant O, Grazianil M and Gaspar T (2004) Effect of NaCl and mannitol iso-osmotic stresses on praline and free polyamine levels in embryogenic Fraxinus angustifolia callus. J. Plant Physiol. 161: 701-708.

Vanishree M, Lee CY, Lo SF, Nalawade SM, Lin CY and Tsay SH (2004) Studies on the production of some important metabolites from medicinal plants by plant tissue cultures. Bot. Bull. Acad. Sci. 45: 1-22.

Zhao X, Tan HJ, Liu YB, Li XR and Chen GX (2009) Effect of salt stress on growth and osmotic regulation in Thellungiella and Arabidopsis callus. Plant Cell Tiss. Org. Cult. 98: 97-103. 